\title{
Characterisation of methicillin-resistant Staphylococcus aureus from Kuwait hospitals with high-level fusidic acid resistance
}

\author{
E. E. UDO and L. E. JACOB
}

Department of Microbiology, Faculty of Medicine, Kuwait University, Kuwait

\begin{abstract}
Forty-seven fusidic acid- and methicillin-resistant Staphylococcus aureus isolates from clinical samples in four hospitals in Kuwait were studied for their relatedness by biotyping and pulsed-field gel electrophoresis (PFGE) and for the genetic location of their resistance determinants. Forty-four isolates were resistant to gentamicin, kanamycin and neomycin. Forty-one isolates were resistant to erythromycin and trimethoprim, 10 were resistant to chloramphenicol and four were resistant to ciprofloxacin. They contained two or three plasmids of $c$. $28,2.8$ and $1.8 \mathrm{~kb}$. Genetic studies demonstrated that resistance to cadmium, propamidine isethionate and ethidium bromide were linked and were carried on the $c$. 28-kb plasmid. Chloramphenicol resistance was encoded by the 2.8 -kb plasmid in resistant isolates. No resistance was associated with the 1.8-kb plasmid and this was considered to be a cryptic plasmid. Resistance to fusidic acid, methicillin, benzylpenicillin, gentamicin, kanamycin, neomycin, tetracycline, trimethoprim, erythromycin and ciprofloxacin were located on the chromosome. All the isolates produced urease, but varied in the production of haemolysins, pigments, lipase and lecithinase and were classified into nine biotypes. In contrast, PFGE divided the isolates into two major patterns with one PFGE type constituting the majority of isolates in all four hospitals. The presence of the dominant PFGE pattern in all four hospitals suggests that it is an epidemic MRSA clone with the capacity to spread. Infection control measures should be directed towards restricting the further spread of this clone.
\end{abstract}

\section{Introduction}

Fusidic acid is active against many gram-positive bacteria, including many anaerobes, but gram-negative bacteria are generally resistant [1-3]. Its minimum inhibitory concentration (MIC) against Staphylococcus aureus and $S$. epidermidis is very low, often between 0.03 and $0.25 \mathrm{mg} / \mathrm{L}$, and it exerts a bactericidal rather than bacteriostatic action [1]. It is extremely active against methicillin-resistant $S$. aureus (MRSA) and $S$. epidermidis $[4,5]$ and is, therefore, a valuable agent in the management of patients with staphylococcal infections including orthopaedic infections [3], acute and chronic osteomyelitis [6] and may be important in the treatment of MRSA endocarditis [2, 3, 7].

Fusidic acid-resistant $S$. aureus can be obtained readily

Received 30 March 1999; revised version received 6 Sept. 1999; accepted 16 Oct. 1999.

Corresponding author: Dr E. E. Udo (e-mail: EDET@hsc. kuniv.edu.kw). in the laboratory by growing the organism in increasing concentrations of the antibiotic [2, 8,9]. This type of resistance is usually located on the bacterial chromosome $[8,9]$. Plasmid-mediated resistance to fusidic acid has also been reported in clinical isolates [10-13]. Because fusidic acid is such an important option, other than vancomycin or teicoplanin, for the treatment of MRSA infections, its combination with other antibiotics has been recommended as protection against the emergence of resistance.

Despite the ease with which resistant strains are obtained in vitro, resistance to fusidic acid has remained low amongst clinical isolates world-wide [2, 14-20]. However, in some countries, fusidic acid resistance is increasing amongst MRSA. In Saudi Arabia, fusidic acid resistance was detected in $10 \%$ of MRSA isolated in one hospital [21]. Fusidic acid resistance has increased from $2 \%$ in 1980 to $9 \%$ in 1995 in Western Australia [22] and was detected in $11 \%$ of mupirocin-resistant MRSA isolated in Auckland, New Zealand in 1992 [23]. Fusidic acid resistance 
has also been detected in $18 \%$ of MRSA isolated in Kuwait hospitals between May 1994 and Dec. 1996 (unpublished results). The increase in resistance in these countries is of concern because it could limit the usefulness of fusidic acid as a therapeutic agent against MRSA infections. An increase in the isolation rate of antibiotic multi-resistant bacteria such as MRSA in a hospital ward or unit may be due to the acquisition and spread of an epidemic clone or the development of resistance in genetically unrelated clones in patients treated with the same antibiotic [24]. Each of these scenarios would demand a different approach towards its control. To better understand the epidemiology of the fusidic acid-resistant MRSA isolated in Kuwait, representative isolates were studied by a combination of antibiogram, biotyping and pulsed-field gel electrophoresis (PFGE). The genetic location of the fusidic acid resistance determinant was also determined.

\section{Materials and methods}

\section{MRSA isolates}

Forty-seven MRSA isolates from clinical specimens obtained between March 1994 and Sept. 1995 in four hospitals were studied. Five were from Mubarak $(\mathrm{MBH})$, six from Adan (ADH), 11 from Amiri (AMH) and 25 from Ibn Sina (ISH) hospitals. They were obtained from wound swabs (24), nasal swabs (7), blood (4), ear swabs (2), eye swabs (4), sputa (3), catheter site (1), axilla (1), and groin (1). The isolates were selected on the basis of their resistance to fusidic acid determined by the disk diffusion test from among MRSA submitted for typing to the Staphylococcal Research Laboratory, Department of Microbiology, Faculty of Medicine, Kuwait University. The isolates were identified as $S$. aureus at the clinical microbiology laboratories of the four hospitals by cultural characteristics, colony morphology, positive results for catalase and tube coagulase test with rabbit plasma (Difco Laboratories, Detroit, MI, USA). S. aureus strain WBG1576, carrying the fusidic acid resistance plasmid pUB101, was provided by Professor W. B. Grubb (Perth, Australia).

\section{Antibiotic susceptibility testing}

Susceptibility to antimicrobial agents was determined by the disk diffusion method [25]. The following antimicrobial agents were tested: methicillin $(5 \mu \mathrm{g})$, penicillin $\mathrm{G}(10 \mathrm{U})$, gentamicin $(10 \mu \mathrm{g})$, kanamycin $(30 \mu \mathrm{g})$, neomycin $(30 \mu \mathrm{g})$, streptomycin $(30 \mu \mathrm{g})$, erythromycin $(15 \mu \mathrm{g})$, clindamycin $(2 \mu \mathrm{g})$, chloramphenicol $(30 \mu \mathrm{g})$, tetracycline $(30 \mu \mathrm{g})$, minocycline $(30 \mu \mathrm{g})$, trimethoprim $(5 \mu \mathrm{g})$, fusidic acid $(10 \mu \mathrm{g})$, rifampicin $(5 \mu \mathrm{g})$, ciprofloxacin $(5 \mu \mathrm{g})$, teicoplanin $(30 \mu \mathrm{g})$, vancomycin $(30 \mu \mathrm{g})$, mupirocin $(5 \mu \mathrm{g})$, cadmium acetate $(50 \mu \mathrm{g})$, propamidine isethionate $(50 \mu \mathrm{g})$, ethidium bromide $(60 \mu \mathrm{g})$. MIC to fusidic acid was determined by the agar dilution method [26] and by
Etest strips (AB Biodisk, Solna, Sweden) according to the manufacturer's protocol.

\section{Biotyping}

Biotyping was performed as described by HamiltonMiller and Maple [27]. The isolates were tested for lecithinase, lipase, haemolysin and pigment production. All test plates were inoculated with MRSA strains with a multi-point inoculator (Sigma).

Lecithinase. Lecithinase was detected on Egg Yolk Agar plates (Egg Yolk Emulsion, Oxoid, 10\% v/v, $\mathrm{NaC} 11 \% \mathrm{w} / \mathrm{v}$ and glucose $1 \%$ in nutrient agar) by opacity surrounding bacterial growth after incubation for $48 \mathrm{~h}$ at $35^{\circ} \mathrm{C}$.

Lipase. This was detected by observing opalescent zones around bacterial growth on nutrient agar plates containing Tween 80 (Fischer Chemical, NJ, USA) 1\% after $48 \mathrm{~h}$ at $35^{\circ} \mathrm{C}$.

Pigment. Evaporated milk (Difco) $20 \% \mathrm{v} / \mathrm{v}$ was added to nutrient agar. The colour of bacterial growth was recorded as white $(\mathrm{W})$, cream $(\mathrm{Cr})$ or Orange (Or) after incubation for $18 \mathrm{~h}$ at $35^{\circ} \mathrm{C}$ and standing at room temperature for a further $18 \mathrm{~h}$.

Haemolysin. Plates consisting of whole sheep blood $5 \%$ in IsoSensitest agar layered on IsoSensitest agar were read after incubation for $24 \mathrm{~h}$ at $35^{\circ} \mathrm{C}$. Strains were classified as haemolytic if lysis occurred either under or around the bacterial growth.

Scoring. A single digit code was obtained by scoring haemolysis as 1 , lipase production as 2 and lecithinase as 4 . The appropriate letter for pigment production was then added. For example, isolate 234 (haemolytic, lecithinase positive and producing cream pigment) was coded as $5 \mathrm{Cr}$.

Urease production. This was detected on Christensen's urea agar slants.

\section{Genetic analysis}

Plasmids were isolated and separated by agarose gel electrophoresis as described previously [28]. Location of resistance determinants was determined by curing and transfer experiments. Plasmid transfer was performed by conjugation and mixed-culture transfer experiments as described previously [29].

\section{PFGE}

Cells were grown overnight in tryptic soy broth and used to prepare agarose blocks according to a method described previously [29]. The formed blocks were incubated in $1.0 \mathrm{ml}$ of EC buffer for $3 \mathrm{~h}$ followed by overnight incubation at $50^{\circ} \mathrm{C}$ in proteinase $\mathrm{K}$ buffer 
(0.5 M EDTA, $5 \mathrm{~mm}$ Tris, sodium laurylsarcosine $1 \%$, $\mathrm{pH} 7.5$ ) containing proteinase $\mathrm{K} 1 \mathrm{mg} / \mathrm{ml}$. The blocks were then washed in $50 \mathrm{mM}$ EDTA for $2 \mathrm{~h}$, changing the buffer after every $30 \mathrm{~min}$, and stored at $4^{\circ} \mathrm{C}$ in $50 \mathrm{~mm}$ EDTA. The blocks were digested with Sma I (Gibco BRL, Gaithersburg MD, USA) according to the manufacturer's instructions. Electrophoresis was performed with agarose $1 \%$ gel (pulsed-field grade, BioRad Laboratories, Hercules, CA, USA) in $0.5 \times \mathrm{TBE}$ buffer with a CHEF DR III apparatus (BioRad) at $14^{\circ} \mathrm{C}$. Linear ramp of switch times was $0.5-40 \mathrm{~s}$. The gels were stained in ethidium bromide $0.5 \mathrm{mg} / \mathrm{L}$ and photographed under UV illumination. Chromosomal patterns were examined and compared by eye and assigned to PFGE types. The relatedness of the strains was determined according to the recommendation of Tenover et al. [30].

\section{Results}

\section{Resistance to antimicrobial agents}

Fusidic acid resistance was detected in $18 \%$ of MRSA from four hospitals in Kuwait between March 1994 and Sept. 1996, but not in any methicillin-susceptible $S$. aureus isolated in the same hospitals at the same time. The 47 isolates studied were obtained from 37 patients (Table 1). The MIC of fusidic acid was initially determined by the agar dilution method with media containing fusidic acid at dilutions from 0.25 to $64 \mathrm{mg} / \mathrm{L}$. After incubation for $18 \mathrm{~h}, 45$ of the 47 MRSA isolates grew on media containing fusidic acid $64 \mathrm{mg} / \mathrm{L}$. This was interpreted as indicating that they had an MIC $>64 \mathrm{mg} / \mathrm{L}$. One isolate (IS201) had an MIC of $16 \mathrm{mg} / \mathrm{L}$ and another isolate (IS32) had an MIC of $32 \mathrm{mg} / \mathrm{L}$. S. aureus strain ATCC $25923 \mathrm{had}$ an MIC of $0.25 \mathrm{mg} / \mathrm{L}$ whereas strain WBG1576, harbouring the fusidic acid resistance plasmid, pUB101, had an MIC of $16 \mathrm{mg} / \mathrm{L}$. The MIC determination was repeated with Etest strips so as to determine the exact MIC value for the isolates. The results demonstrated that all the 45 isolates with $\mathrm{MICs}>64 \mathrm{mg} / \mathrm{L}$ by the agar dilution method had MICs $>256 \mathrm{mg} / \mathrm{L}$ by the Etest. MIC values with the Etest for strains ATCC25923, WBG1576, IS32 and IS201 were the same as those obtained by the agar incorporation method.

With regard to resistance to other antimicrobial agents, all isolates were resistant to penicillin $G$, cadmium, propamidine isethionate and ethidium bromide. Forty four isolates were resistant to gentamicin, kanamycin and neomycin. Forty-two isolates were resistant to streptomycin; 42 were also resistant to tetracycline; 43 were resistant to erythromycin and 40 were resistant to trimethoprim. Chloramphenicol resistance was detected in 10 isolates and only four isolates were resistant to ciprofloxacin. They were all susceptible to vancomycin, teicoplanin, rifampicin, minocycline and mupirocin.

\section{Fusidic acid consumption}

A review of pharmacy records in the hospitals showed that oral fusidic acid was used in two of the hospitals. Surprisingly, there was no record of fusidic acid use in the burns unit where most of the fusidic acid-resistant isolates originated; however, topical fusidic acid was used in other areas of the same hospital. An average of $650 \mathrm{~g}$ of oral fusidic acid was consumed annually in the two hospitals. No data for fusidic acid consumption were available for one of the hospitals.

\section{Plasmid analysis of isolates}

Two major plasmid patterns were observed (Fig. 1). One type, consisting of 37 isolates, harboured three plasmids of 28, c. 2.8 and $1.8 \mathrm{~kb}$. This was designated plasmid type A (Table 1). The second type, designated plasmid type B, consisted of eight isolates that harboured two plasmids of c. 28 and $2.8 \mathrm{~kb}$. Two isolates had different plasmid profiles (not shown). These were isolate numbers 359 (which harboured a single plasmid of c. $40 \mathrm{~kb}$ ) and 436 (which harboured two plasmids of c. 28 and $1.8 \mathrm{~kb}$ ).

To determine whether the genes for fusidic acid resistance in these isolates resided on a plasmid or on the chromosome, six representative isolates (212, 146, 358, IS20, 428 and 437) from the two main plasmid patterns were studied by curing and transfer experiments. In curing experiments, resistance to cadmium, propamidine isethionate and ethidium bromide were lost together and their loss was accompanied by the loss of a plasmid of $c .28 \mathrm{~kb}$ in all six isolates. Similarly, chloramphenicol resistance was lost on curing from isolates IS20 and 437 and its loss was accompanied by the loss of a $2.8-\mathrm{kb}$ plasmid. In transfer experiments, no resistance determinants were transferred by conjugation, indicating that they did not harbour conjugative plasmids. However, resistance to cadmium, propamidine isethionate and ethidium bromide were co-transferred with a 28 -kb plasmid from all six isolates in mixed-culture transfer experiments. Similarly, the transfer of chloramphenicol resistance in mixed-culture transfer experiments was accompanied by the transfer of a $2.8-\mathrm{kb}$ plasmid from isolates 428 and 437. There was no transfer of fusidic acid resistance from any of the six isolates. The six $28-\mathrm{kb}$ plasmids encoding resistance to cadmium, propamidine isethionate and ethidium bromide were digested with EcoRI restriction endonuclease and their restriction patterns were compared. They all had identical EcoRI restriction patterns; the sizes were 11.2, 6.7, 4.2, 2.5, 2.3 and $1.3(28.2) \mathrm{kb}$.

\section{Biotyping of isolates}

The biotypes obtained are summarised in Table 1. All the isolates produced urease and the majority (33 of 47) produced a haemolysin that lysed sheep red blood 
Table 1. Properties of fusidic acid-resistant MRSA isolates

\begin{tabular}{|c|c|c|c|c|c|c|c|c|c|c|c|c|c|c|}
\hline \multirow{2}{*}{$\begin{array}{l}\text { Patient } \\
\text { no. }\end{array}$} & \multirow{2}{*}{$\begin{array}{l}\text { Isolate } \\
\text { no. }\end{array}$} & \multirow[b]{2}{*}{ Hospital } & \multirow[b]{2}{*}{ Date* } & \multirow[b]{2}{*}{ Ward } & \multirow[b]{2}{*}{ Specimen } & \multicolumn{6}{|c|}{ Antibiotic resistance } & \multirow[b]{2}{*}{ Biotype } & \multirow{2}{*}{$\begin{array}{c}\text { Plasmid } \\
\text { types }\end{array}$} & \multirow{2}{*}{$\begin{array}{l}\text { PFGE } \\
\text { types }\end{array}$} \\
\hline & & & & & & $\mathrm{Gm}$ & Em & $\mathrm{Cm}$ & Tet & Cip & Tmp & & & \\
\hline 1 & 133 & $\mathrm{MBH}$ & $8 / 94$ & MM & Wound & $\mathrm{R}$ & $\mathrm{R}$ & $\mathrm{S}$ & $\mathrm{R}$ & $\mathrm{S}$ & $\mathrm{R}$ & $5 \mathrm{Cr}$ & A & I \\
\hline 2 & 134 & MBH & $8 / 94$ & MM & Lung Abscess & $\mathrm{R}$ & $\mathrm{R}$ & $\mathrm{S}$ & $\mathrm{R}$ & $\mathrm{S}$ & $\mathrm{R}$ & $5 \mathrm{Cr}$ & A & $\mathrm{Ib}$ \\
\hline 3 & 357 & MBH & $2 / 95$ & ICU & $\mathrm{N} / \mathrm{swab}$ & $\mathrm{R}$ & $\mathrm{R}$ & $\mathrm{S}$ & $\mathrm{R}$ & $\mathrm{s}$ & $\mathrm{R}$ & $10 r$ & A & I \\
\hline 3 & 358 & MBH & $2 / 95$ & ICU & Axilla & $\mathrm{R}$ & $\mathrm{R}$ & $\mathrm{S}$ & $\mathrm{R}$ & $\mathrm{S}$ & $\mathrm{R}$ & $1 \mathrm{Or}$ & A & I \\
\hline 3 & 359 & MBH & $2 / 95$ & ICU & Groin & $\mathrm{R}$ & $\mathrm{R}$ & $\mathrm{S}$ & $\mathrm{R}$ & $\mathrm{S}$ & $\mathrm{R}$ & $1 \mathrm{Or}$ & A & I \\
\hline 4 & 212 & $\mathrm{ADH}$ & $9 / 95$ & ICU & Wound & $\mathrm{R}$ & $\mathrm{R}$ & $\mathrm{S}$ & $\mathrm{R}$ & $\mathrm{S}$ & $\mathrm{R}$ & $10 r$ & A & $\mathrm{Ib}$ \\
\hline 5 & 434 & $\mathrm{ADH}$ & $9 / 95$ & $\mathrm{ICU}$ & Blood & $\mathrm{R}$ & $\mathrm{R}$ & $\mathrm{S}$ & $\mathrm{R}$ & $\mathrm{s}$ & $\mathrm{R}$ & $2 \mathrm{Or}$ & A & I \\
\hline 5 & 435 & $\mathrm{ADH}$ & $9 / 95$ & $\mathrm{ICU}$ & Wound & $\mathrm{R}$ & $\mathrm{R}$ & $\mathrm{S}$ & $\mathrm{R}$ & $\mathrm{S}$ & $\mathrm{R}$ & $3 \mathrm{Or}$ & A & I \\
\hline 6 & 436 & $\mathrm{ADH}$ & $9 / 95$ & $\mathrm{ICU}$ & Ear swab & $\mathrm{R}$ & $\mathrm{R}$ & $\mathrm{S}$ & $\mathrm{R}$ & $\mathrm{S}$ & $\mathrm{R}$ & $0 \mathrm{Or}$ & $\mathrm{C}$ & I \\
\hline 7 & 437 & $\mathrm{ADH}$ & $9 / 95$ & ICU & Wound & $\mathrm{R}$ & $\mathrm{R}$ & $\mathrm{R}$ & $\mathrm{R}$ & $\mathrm{S}$ & $\mathrm{R}$ & $3 \mathrm{Or}$ & B & I \\
\hline 7 & 438 & $\mathrm{ADH}$ & $9 / 95$ & $\mathrm{ICU}$ & Sputum & $\mathrm{R}$ & $\mathrm{R}$ & $\mathrm{S}$ & $\mathrm{R}$ & $\mathrm{S}$ & $\mathrm{R}$ & $3 \mathrm{Or}$ & A & I \\
\hline 8 & 397 & AMH & $7 / 95$ & ICU & $\mathrm{N} / \mathrm{swab}$ & $\mathrm{R}$ & $\mathrm{R}$ & $\mathrm{S}$ & $\mathrm{R}$ & $\mathrm{S}$ & $\mathrm{R}$ & $5 \mathrm{Or}$ & A & I \\
\hline 8 & 398 & $\mathrm{AMH}$ & $7 / 95$ & ICU & $\mathrm{C}^{\prime} /$ site & $\mathrm{R}$ & $\mathrm{R}$ & $\mathrm{S}$ & $\mathrm{R}$ & $\mathrm{S}$ & $\mathrm{R}$ & $5 \mathrm{Or}$ & A & I \\
\hline 9 & 399 & AMH & $7 / 95$ & ICU & Blood & $\mathrm{R}$ & $\mathrm{R}$ & $\mathrm{S}$ & $\mathrm{R}$ & $\mathrm{S}$ & $\mathrm{R}$ & $10 r$ & A & I \\
\hline 10 & 400 & AMH & $7 / 95$ & $\mathrm{ICU}$ & $\mathrm{N} / \mathrm{swab}$ & $\mathrm{R}$ & $\mathrm{R}$ & $\mathrm{R}$ & $\mathrm{R}$ & $\mathrm{S}$ & $\mathrm{R}$ & $5 \mathrm{Or}$ & B & I \\
\hline 11 & 411 & $\mathrm{AMH}$ & $7 / 95$ & ICU & Eye swab & $\mathrm{R}$ & $\mathrm{R}$ & $\mathrm{R}$ & $\mathrm{R}$ & $\mathrm{S}$ & $\mathrm{R}$ & $5 \mathrm{Or}$ & B & I \\
\hline 12 & 413 & AMH & $7 / 95$ & ICU & N/swab & $\mathrm{R}$ & $\mathrm{R}$ & $\mathrm{S}$ & $\mathrm{R}$ & $\mathrm{S}$ & $\mathrm{R}$ & $10 r$ & A & I \\
\hline 12 & 419 & AMH & $7 / 95$ & $\mathrm{ICU}$ & Blood & $\mathrm{R}$ & $\mathrm{R}$ & $\mathrm{S}$ & $\mathrm{R}$ & $\mathrm{S}$ & $\mathrm{R}$ & $5 \mathrm{Or}$ & A & I \\
\hline 13 & 421 & AMH & $7 / 95$ & ICU & Bed sore & $\mathrm{R}$ & $\mathrm{R}$ & $\mathrm{R}$ & $\mathrm{R}$ & $\mathrm{S}$ & $\mathrm{R}$ & $5 \mathrm{Or}$ & B & I \\
\hline 13 & 423 & AMH & $7 / 95$ & $\mathrm{ICU}$ & $\mathrm{N} /$ swab & $\mathrm{R}$ & $\mathrm{R}$ & $\mathrm{R}$ & $\mathrm{R}$ & $\mathrm{S}$ & $\mathrm{R}$ & $5 \mathrm{Or}$ & B & I \\
\hline 14 & 428 & AMH & $7 / 95$ & ICU & $\mathrm{N} / \mathrm{swab}$ & $\mathrm{R}$ & $\mathrm{S}$ & $\mathrm{R}$ & $\mathrm{R}$ & $\mathrm{S}$ & $\mathrm{R}$ & $5 \mathrm{Or}$ & B & I \\
\hline 15 & 430 & AMH & $7 / 95$ & ICU & $\mathrm{N} /$ swab & $\mathrm{R}$ & $\mathrm{R}$ & $\mathrm{S}$ & $\mathrm{R}$ & $\mathrm{S}$ & $\mathrm{R}$ & $4 \mathrm{Cr}$ & A & I \\
\hline 16 & 146 & ISH & $4 / 94$ & ICU & Ear swab & $\mathrm{R}$ & $\mathrm{R}$ & $\mathrm{S}$ & $\mathrm{R}$ & $\mathrm{S}$ & $\mathrm{R}$ & $1 \mathrm{Or}$ & A & Id \\
\hline 17 & IS4 & ISH & $3 / 94$ & Burn & Wound & $\mathrm{R}$ & $\mathrm{R}$ & $\mathrm{S}$ & $\mathrm{R}$ & $\mathrm{S}$ & $\mathrm{R}$ & $1 \mathrm{Or}$ & A & I \\
\hline 17 & IS5 & ISH & $3 / 94$ & Burn & Blood & $\mathrm{R}$ & $\mathrm{R}$ & $\mathrm{S}$ & $\mathrm{R}$ & $\mathrm{S}$ & $\mathrm{R}$ & $1 \mathrm{Or}$ & A & Ia \\
\hline 18 & IS7 & ISH & $3 / 94$ & Burn & Wound & $\mathrm{R}$ & $\mathrm{R}$ & $\mathrm{S}$ & $\mathrm{R}$ & $\mathrm{S}$ & $\mathrm{R}$ & $1 \mathrm{Or}$ & A & I \\
\hline 18 & IS8 & ISH & $3 / 94$ & Burn & Eye swab & $\mathrm{R}$ & $\mathrm{R}$ & $\mathrm{S}$ & $\mathrm{R}$ & $\mathrm{S}$ & $\mathrm{R}$ & $1 \mathrm{Or}$ & B & I \\
\hline 19 & IS15 & ISH & $7 / 94$ & OPD & Wound & $\mathrm{R}$ & $\mathrm{R}$ & $\mathrm{S}$ & $\mathrm{R}$ & $\mathrm{S}$ & $\mathrm{R}$ & $3 \mathrm{Or}$ & B & Ia \\
\hline 20 & IS19 & ISH & $6 / 94$ & OPD & Wound & $\mathrm{R}$ & $\mathrm{R}$ & $\mathrm{S}$ & $\mathrm{R}$ & $\mathrm{S}$ & $\mathrm{R}$ & $3 \mathrm{Or}$ & A & I \\
\hline 21 & IS20 & ISH & $6 / 94$ & Burn & Wound & $\mathrm{R}$ & $\mathrm{R}$ & $\mathrm{R}$ & $\mathrm{R}$ & $\mathrm{S}$ & $\mathrm{R}$ & $1 \mathrm{Or}$ & A & I \\
\hline 22 & IS21 & ISH & $8 / 94$ & Burn & Sputum & $\mathrm{R}$ & $\mathrm{R}$ & $\mathrm{R}$ & $\mathrm{R}$ & $\mathrm{S}$ & $\mathrm{R}$ & $3 \mathrm{Or}$ & A & I \\
\hline 23 & IS22 & ISH & $8 / 94$ & Burn & Wound & $\mathrm{R}$ & $\mathrm{R}$ & $\mathrm{S}$ & $\mathrm{R}$ & $\mathrm{S}$ & $\mathrm{R}$ & $3 \mathrm{Or}$ & A & II \\
\hline 24 & IS27 & ISH & $4 / 94$ & Burn & Wound & $\mathrm{R}$ & $\mathrm{R}$ & $\mathrm{S}$ & $\mathrm{R}$ & $\mathrm{S}$ & $\mathrm{R}$ & $0 \mathrm{Or}$ & A & II \\
\hline 25 & IS31 & ISH & $4 / 94$ & Burn & Wound & $\mathrm{R}$ & $\mathrm{R}$ & $\mathrm{S}$ & $\mathrm{R}$ & $\mathrm{S}$ & $\mathrm{R}$ & $0 \mathrm{Or}$ & A & II \\
\hline 26 & IS32 & ISH & $4 / 94$ & Burn & Sputum & $\mathrm{R}$ & $\mathrm{R}$ & $\mathrm{R}$ & $\mathrm{S}$ & $\mathrm{S}$ & $\mathrm{S}$ & $0 \mathrm{Or}$ & A & II \\
\hline 27 & IS191 & ISH & $4 / 94$ & ICU & Wound & $\mathrm{R}$ & $\mathrm{R}$ & $\mathrm{S}$ & $\mathrm{R}$ & $\mathrm{S}$ & $\mathrm{R}$ & $0 \mathrm{Or}$ & A & II \\
\hline 28 & IS198 & ISH & $4 / 94$ & ICU & Sputum & $\mathrm{R}$ & $\mathrm{R}$ & $\mathrm{S}$ & $\mathrm{R}$ & $\mathrm{S}$ & $\mathrm{R}$ & $0 \mathrm{Or}$ & A & II \\
\hline 29 & IS201 & ISH & $4 / 94$ & Burn & Eye swab & $\mathrm{R}$ & $\mathrm{R}$ & $\mathrm{R}$ & $\mathrm{R}$ & $\mathrm{R}$ & $\mathrm{S}$ & $0 \mathrm{Or}$ & A & II \\
\hline 30 & IS221 & ISH & $4 / 94$ & Burn & Wound & $\mathrm{R}$ & $\mathrm{R}$ & $\mathrm{S}$ & $\mathrm{R}$ & $\mathrm{R}$ & $\mathrm{S}$ & $0 \mathrm{Or}$ & A & II \\
\hline 31 & IS222 & ISH & $4 / 94$ & ICU & Wound & $\mathrm{R}$ & $\mathrm{S}$ & $\mathrm{S}$ & $\mathrm{S}$ & $\mathrm{S}$ & $\mathrm{R}$ & $0 \mathrm{Or}$ & A & II \\
\hline 32 & IS223 & ISH & $4 / 94$ & OPD & Wound & $\mathrm{S}$ & $\mathrm{R}$ & $\mathrm{S}$ & $\mathrm{S}$ & $\mathrm{S}$ & $\mathrm{S}$ & $0 \mathrm{Or}$ & A & Ic \\
\hline 33 & IS226 & ISH & $4 / 94$ & ICU & Wound & $\mathrm{R}$ & $\mathrm{R}$ & $\mathrm{S}$ & $\mathrm{R}$ & $\mathrm{R}$ & $\mathrm{S}$ & $3 \mathrm{Or}$ & A & I \\
\hline 34 & IS227 & ISH & $7 / 94$ & ICU & Wound & $\mathrm{R}$ & $\mathrm{R}$ & $\mathrm{S}$ & $\mathrm{S}$ & $\mathrm{S}$ & $\mathrm{R}$ & $3 \mathrm{Cr}$ & A & I \\
\hline 34 & IS228 & ISH & $7 / 94$ & ICU & Wound & $\mathrm{R}$ & $\mathrm{S}$ & $\mathrm{S}$ & $\mathrm{R}$ & $\mathrm{R}$ & $\mathrm{R}$ & $0 \mathrm{Or}$ & A & II \\
\hline 35 & IS231 & ISH & $8 / 94$ & ICU & Wound & $\mathrm{R}$ & $\mathrm{S}$ & $\mathrm{S}$ & $\mathrm{R}$ & $\mathrm{S}$ & $\mathrm{R}$ & $0 \mathrm{Or}$ & A & I \\
\hline 36 & 370 & ISH & $4 / 94$ & ICU & Eye swab & $\mathrm{S}$ & $\mathrm{R}$ & $\mathrm{S}$ & $\mathrm{R}$ & $\mathrm{S}$ & $\mathrm{R}$ & $5 \mathrm{Or}$ & A & I \\
\hline 37 & 374 & ISH & $4 / 94$ & ICU & Blood & $\mathrm{S}$ & $\mathrm{R}$ & $\mathrm{S}$ & $\mathrm{S}$ & $\mathrm{S}$ & $\mathrm{S}$ & $5 \mathrm{Or}$ & A & I \\
\hline
\end{tabular}

MBH, Mubarak hospital; ADH, Adan hospital; AMH, Amiri hospital; ISH, Ibn Sina hospital. All the isolates had fusidic acid MIC $>256 \mathrm{mg} / \mathrm{L}$ except two isolates, IS32 (MIC $32 \mathrm{mg} / \mathrm{L}$ ) and IS201 (MIC $16 \mathrm{mg} / \mathrm{L}$ ).

MM, male medical; N/swab, nasal swab; $\mathrm{C} /$ site, catheter site.

${ }^{*}$ Date of receipt of isolates.

cells. Thirteen isolates produced lecithinase and nine produced lipase. On the basis of these properties they were grouped into nine biotypes. The most common biotype was 1 Or (12 isolates, orange pigment and haemolysin) followed by $5 \mathrm{Or}$ (10 isolates, orange pigment, lecithinase and haemolysin), 0Or (10 isolates, orange pigment, no lecithinase, lipase or haemolysin), 3Or ( 8 isolates, orange pigment, lipase and haemoly$\sin ), 5 \mathrm{Cr}$ ( 2 isolates, cream pigment, lecithinase and haemolysin), $0 \mathrm{Cr}$ ( 2 isolates, cream pigment), $4 \mathrm{Cr}$ (1 isolate, cream pigment and lecithinase), 2Or (1 isolate, orange pigment and lipase) and $3 \mathrm{Cr}$ (1 isolate, cream pigment, lipase and haemolysin).

\section{PFGE of MRSA isolates}

Two major PFGE patterns were obtained, as summarised in Table 1. The majority of the fusidic acidresistant MRSA isolates belonged to PFGE pattern type 1. The five isolates from MBH had two PFGE patterns; four of them had the type-I pattern. The fifth isolate differed from the other four isolates lacking three bands but was considered to be related to them and was designated as PFGE type 1b (Fig. 2, lane B). The six isolates from $\mathrm{ADH}$ also belonged to two PFGE patterns; five of them had the type I pattern and one had the type Ib pattern (Fig. 2). The 11 isolates from 


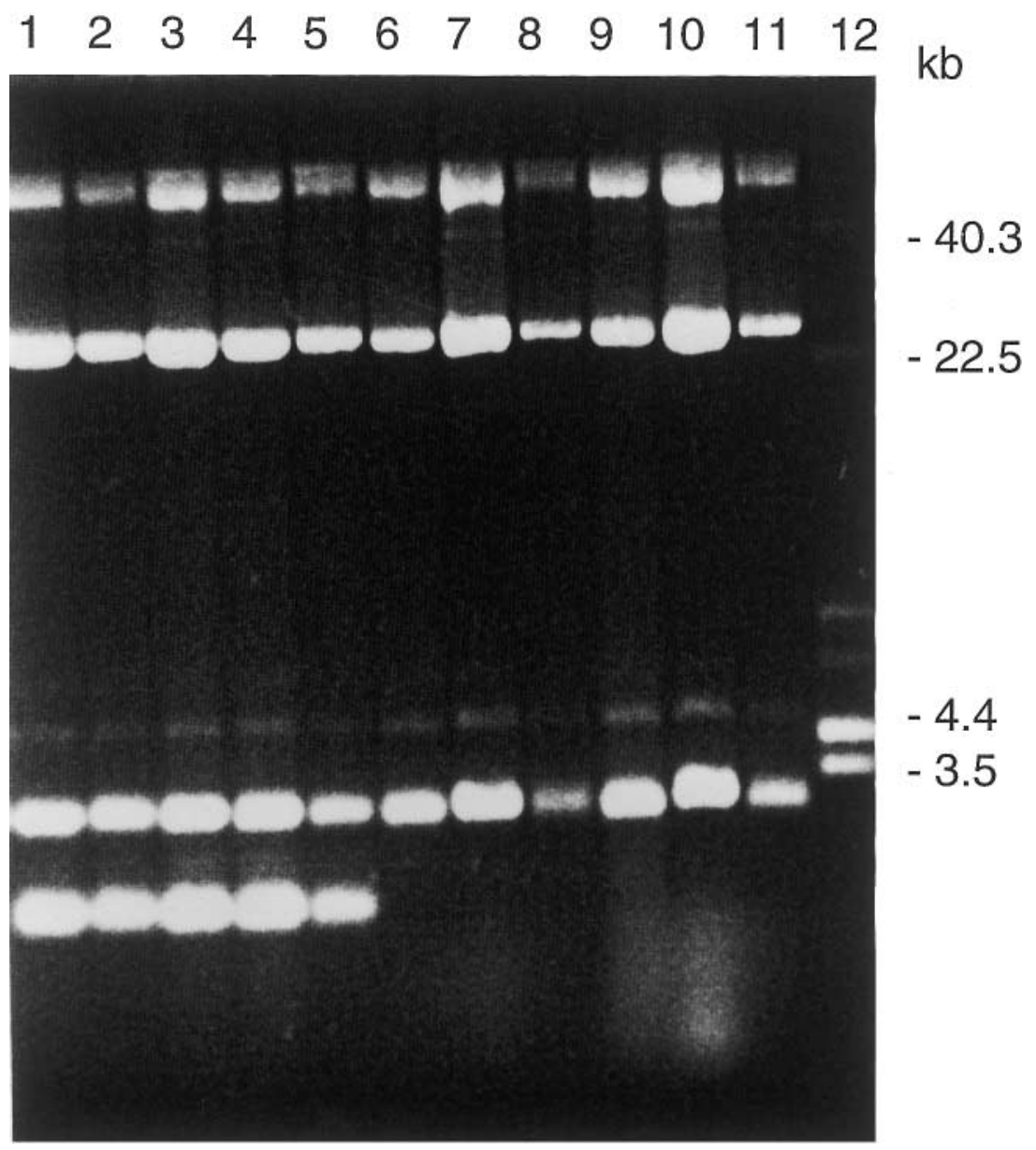

Fig. 1. Plasmid contents of representative fusidic acid-resistant MRSA. Lanes 1-11, MRSA isolates. Lane 12, plasmid molecular standards. Lanes 1-5, representative isolates containing three plasmids (28, 2.8 and $1.8 \mathrm{~kb})$ : 1, isolate 133; $\mathbf{2}$, isolate $134 ; \mathbf{3}$, isolate $434 ; \mathbf{4}$, isolate $398 ; \mathbf{5}$, isolate IS5. Lanes $\mathbf{6 - 1 1}$, representative isolates containing two plasmids (28 and $2.8 \mathrm{~kb}$ ): $\mathbf{6}$, isolate $437 ; \mathbf{7}$, isolate $400 ; \mathbf{8}$, isolate $411 ; \mathbf{9}$, isolate $428 ; \mathbf{1 0}$, isolate IS18; 11 , isolate IS15.

AMH had identical patterns and were of type I. Isolates from ISH had three PFGE patterns (Fig. 3). Fourteen of the 25 isolates had the PFGE type I pattern, 10 had the type II pattern and were clearly different from those with the type I pattern. Two isolates, IS5 and IS19 (designated type Ia) differed from the rest of the type I by loss of a fragment at the 100-kb region (Fig. 3, lanes $\mathrm{A}$ and E). Isolate 223 (Fig. 3, lane B) also had one band different from the type I pattern and was designated type Ic. Another isolate (no. 146) had four bands different from the type I pattern and was designated as type Id.

\section{Comparison of biotyping and PFGE}

Biotyping distinguished the isolates into nine types with four biotypes, 1Or (12 isolates), 5Or (10 isolates), 0 Or (10 isolates) and $3 \mathrm{Or}$ ( 8 isolates) as the dominant biotypes. In contrast, PFGE distinguished them into two major types with one - type I - and its variants consisting of 37 isolates as the dominant clone. Isolates that had the type 1 PFGE pattern had different biotypes. The 10 isolates that had the type II PFGE pattern were of two biotypes. In some isolates both biotyping and PFGE had concordant results. Both methods identified isolates that had the PFGE type II patterns as clearly distinct from the rest of the isolates. Biotyping also identified the two isolates obtained from patients $1,3,8,13$ and 17 as being the same. In the case of patient 34 , both PFGE and biotyping distinguished the two isolates from this patient as different.

\section{Discussion}

Forty-seven fusidic acid-resistant isolates of MRSA from four hospitals in Kuwait were characterised to determine the genetic location of their resistance determinants and their genetic relatedness. Forty-five of them expressed high levels of fusidic acid resistance (MIC $>256 \mathrm{mg} / \mathrm{L}$ ), which was higher than the fusidic acid resistance MICs of $75-150 \mathrm{mg} / \mathrm{L}$ reported by Chopra [8], $64 \mathrm{mg} / \mathrm{L}$ reported by O'Brien et al. [9] and $16 \mathrm{mg} / \mathrm{L}$ expressed by fusidic acid resistance plasmid pUB101 [9, 10, 13].

The determinants for fusidic acid resistance can be plasmid- $[10,12,13]$ or chromosomally- $[8,9]$ mediated in $S$. aureus. Plasmids encoding resistance to cadmium and penicillinase production $[10,11,13]$ 


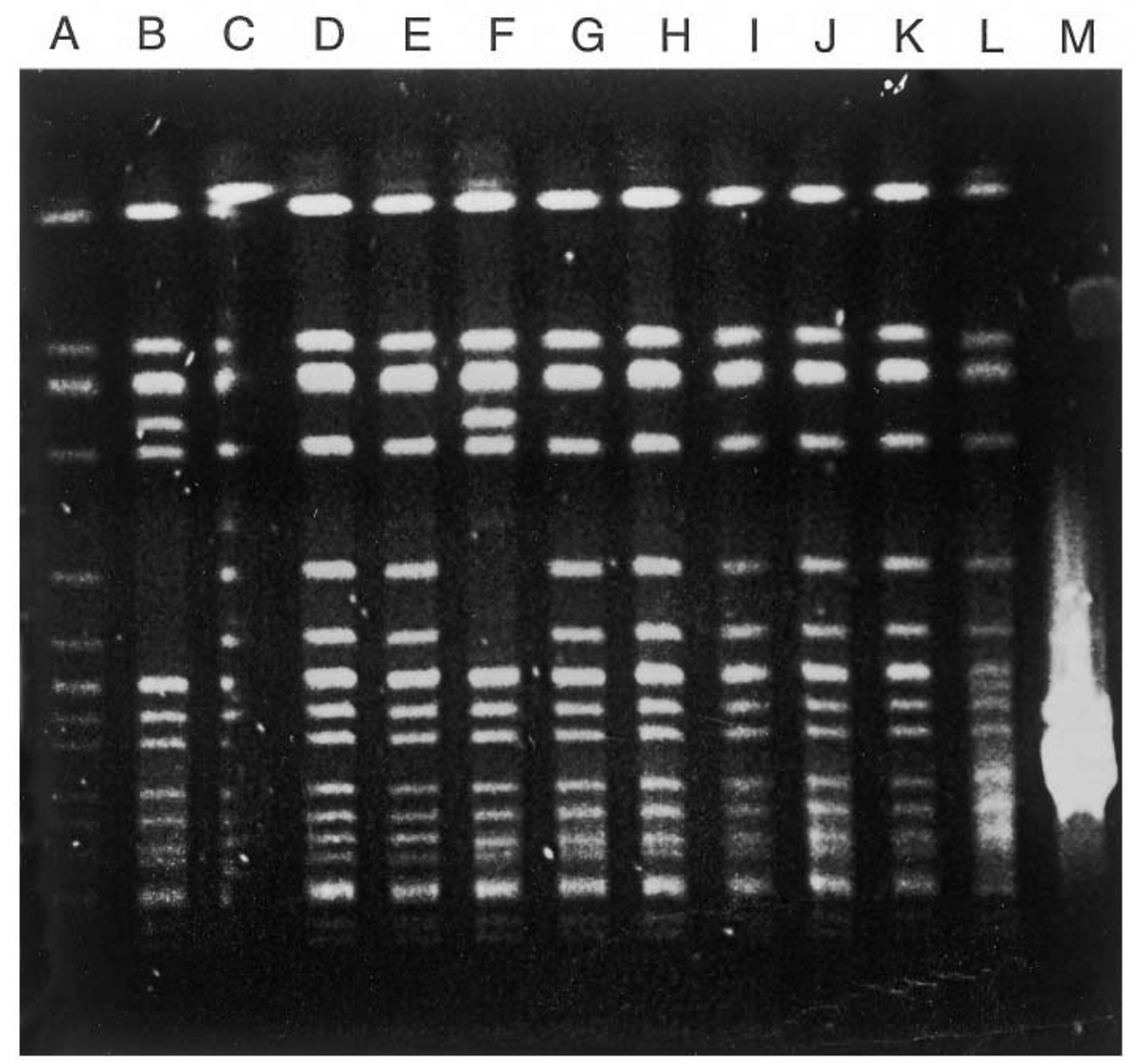

Fig. 2. PFGE patterns of isolates from $\mathrm{MBH}, \mathrm{ADH}$ and $\mathrm{AMH}$ hospitals. Lanes $\mathbf{A}-\mathbf{E}$, isolates from $\mathrm{MBH}$ : $\mathbf{A}$, isolate 133; B (type Ib), isolate 134; C, isolate 357; D, isolate 358; E, isolate 359. Lanes $\mathbf{F}-\mathbf{K}$ : isolates from ADH: F, isolate 212 (type $\mathrm{Ib}$ ); $\mathbf{G}$, isolate 434; $\mathbf{H}$, isolate 435; I, isolate 436; J, isolate 437; K, isolate 438. Lane $\mathbf{L}$, isolate 397, from $\mathrm{AMH}$; the 11 isolates from this hospital had identical PFGE patterns (type I). Lane M, unseparated size marker DNA.

and aminoglycoside resistance have been reported previously [12]. In the present study, fusidic acid resistance was neither lost on curing nor transferred in laboratory experiments. Furthermore, it was not associated with the $28-\mathrm{kb}$ plasmid encoding resistance to cadmium, propamidine isethionate and ethidium bromide in the isolates. These results indicated that fusidic acid resistance was chromosomally located in these isolates. Similarly, resistance to methicillin, benzylpenicillin, gentamicin, kanamycin, neomycin, streptomycin, tetracycline, trimethoprim and ciprofloxacin were chromosomal. The very high MIC values of fusidic acid in these isolates appear to confirm its chromosomal location, as mutation of susceptible $S$. aureus to chromosomal fusidic acid resistance resulted in high fusidic acid MIC values [8,9] and plasmidborne fusidic acid resistance reported previously expressed lower MIC values [9, 10, 13]. However, in contrast to isolates mutated to chromosomal resistance in vitro, which grew more slowly than the parental strain, formed small colonies and reverted to full sensitivity in the absence of fusidic acid $[9,16]$, the isolates in this study grew well, formed large colonies and the resistance was stable when stored for years in the absence of fusidic acid.

Chloramphenicol resistance was located on a $2.8-\mathrm{kb}$ plasmid in the chloramphenicol-resistant isolates tested.
However, chloramphenicol-susceptible isolates also harboured a $2.8-\mathrm{kb}$ plasmid (Table 1, Fig. 1). It would appear that the $2.8-\mathrm{kb}$ plasmid in the chloramphenicolsusceptible isolates had a different resistance phenotype. Erthromycin resistance has been found to be located on a $2.8-\mathrm{kb}$ plasmid in some MRSA isolates from Kuwait hospitals (E. E. Udo, unpublished results).

Epidemiological typing of MRSA to investigate the nature of its spread is a useful tool which can lead to more effective intervention or control strategies $[24,27]$. However, the majority of the methods are complex and technically demanding, which restricts their use to research or reference laboratories [24, 27]. Consequently, Hamilton-Miller and Maple [27] investigated the use of simple and inexpensive low technology typing techniques for the study of $S$. aureus and concluded that biotyping and antibiograms can give rapid and useful information within $24 \mathrm{~h}$ and the tests can be performed in any medical microbiology laboratory. In the present study, biotyping was useful in identifying two isolates obtained from the same patient as identical or different; in this instance it had concordant results with PFGE. This is significant, because the information often required is whether isolates obtained from different sites of the same patient are the same or different. Biotyping also had concordant results with PFGE with regard to identify- 


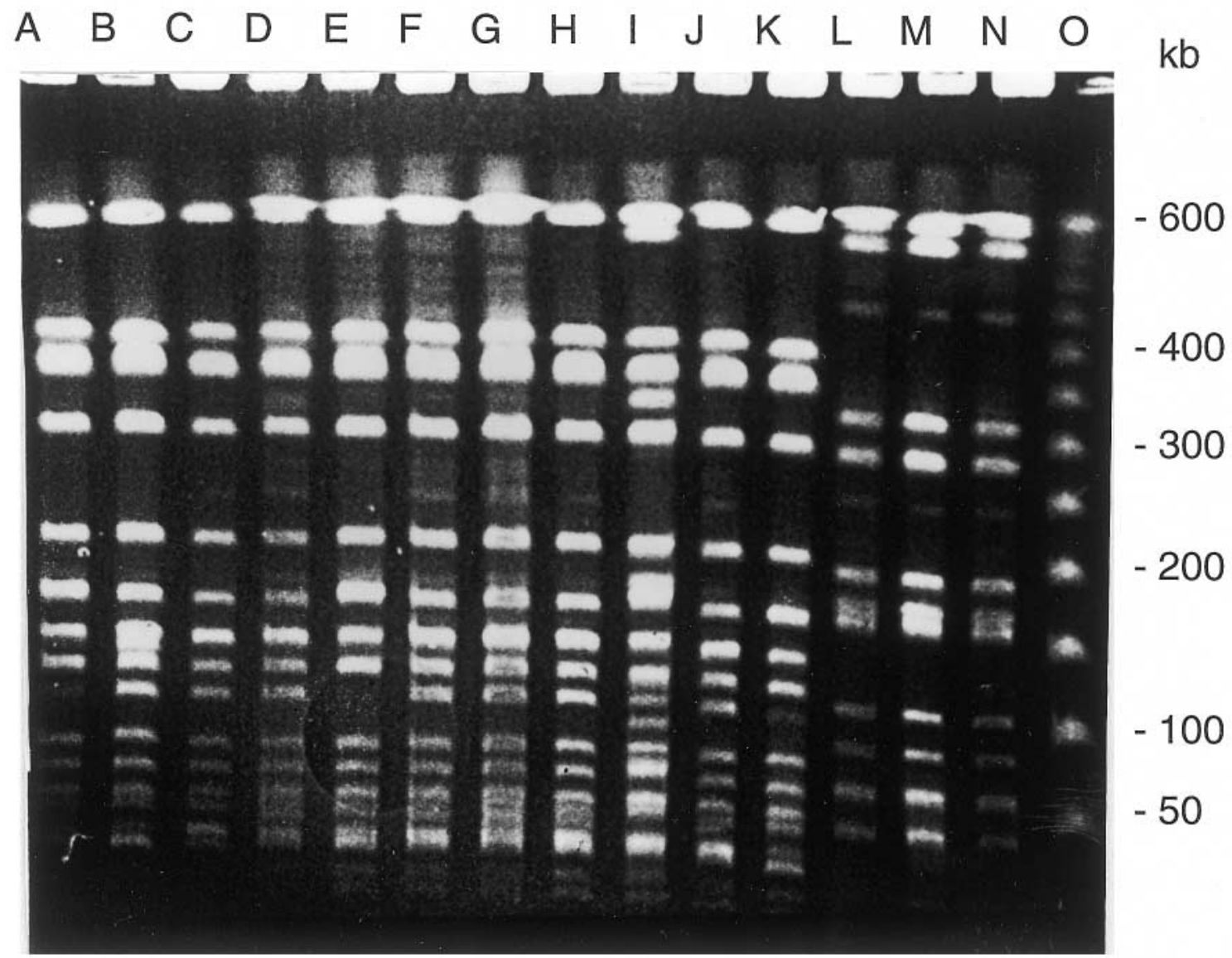

Fig. 3. PFGE patterns of representative isolates from ISH. Lanes $\mathbf{C}, \mathbf{D}, \mathbf{F}, \mathbf{G}, \mathbf{H}, \mathbf{J}, \mathbf{K}$, type I pattern; A, isolate IS5 (type Ia); B, isolate IS223 (type Ic); E, isolate IS15 (type Ia); I, isolate 146 (type Id). Lanes $\mathbf{L}, \mathbf{M}$ and $\mathbf{N}$, representative isolates with PFGE type II pattern: L, isolate IS32; M, isolate 222; N, isolate 228. Lane $\mathbf{O}$, size markers $(\mathrm{Kb})$.

ing isolates with PFGE type II pattern as distinct from the other isolates. On the other hand, whereas biotyping identified nine of the different clones, PFGE revealed two major clones among the isolates. The discrepancy observed between the two typing methods highlights the difficulties encountered in comparing phenotypic and genotypic methods. Although PFGE is considered to be more discriminating than the phenotypic methods, including biotyping, the results of this study also demonstrated that biotyping can be useful and merits a place in routine clinical laboratories, especially those laboratories lacking the benefit of more expensive genotypic methods.

Analysis with PFGE demonstrated that two main clones of fusidic acid-resistant MRSA were present in the four hospitals. One clone (type II) was restricted to one hospital while the other (type I) clone was found in all four hospitals. The presence of the type II clone in only one hospital may indicate that it lacks the capacity to spread and is not an epidemic clone, whereas the type I clone has the capacity to spread. The presence of the same clone of MRSA in different hospitals clearly suggests that it possesses a capacity to spread, which qualifies it as an epidemic MRSA clone. The spread of the type I clone among the four hospitals may have resulted from the transfer of patients between them. Two of the hospitals, MBH and AMH, are parts of the teaching hospital complex of the Kuwait University Medical School and patients are often transferred between them. Also, the burns unit at ISH is the only such facility in the country and patients are often transferred between it and other hospitals, providing excellent opportunity for the transfer of resistant organisms between the hospitals. Also, it is interesting that most of the isolates were from patients residing in the ICU or the burns unit, areas that often accept patients transferred from other hospitals. The survival of this clone in these wards over time would be aided by antibiotic use in them.

The expression of high levels of fusidic acid in the different clones noted here may indicate that they either acquired fusidic acid resistance independently or as a result of horizontal transfer. Although fusidic acid resistance could not be transferred in vitro, this does not exclude the possibility of such transfer occurring in nature. The failure to transfer it in the laboratory may reflect the limitations of current techniques.

The finding that the presence of fusidic acid-resistant MRSA in this study was due to the circulation of a few 
clones, with the epidemic clone spreading in all four hospitals is a positive finding, because it suggests that if the spread of the epidemic clone is controlled, the number of fusidic acid-resistant MRSA in these hospitals would be small and would be comparable to that seen in the UK [17] and Denmark [14], where fusidic acid resistance among $S$. aureus has remained low despite its use for many years. Therefore, infection control efforts should be directed towards restricting the spread of the fusidic acid-resistant MRSA epidemic clone in these hospitals.

This study was supported by grant MI 091 from Kuwait University Research Administration. We thank Professor W. B. Grubb for the gift of plasmid pUB101 and Mrs Bindu Matthews for help with plasmid isolation.

\section{References}

1. Verbist L. The antimicrobial activity of fusidic acid. J Antimicrob Chemother 1990; 25 Suppl B: 1-5.

2. Lambert HP, O'Grady FW. Antibiotic and chemotherapy, 6th edn. London, Churchill Livingstone 1992: 157-159.

3. Portier H. A multicentre, open, clinical trial of a new intravenous formulation of fusidic acid in severe staphylococcal infections. J Antimicrob Chemother 1990; 25 Suppl B: 39-44.

4. Huebner J, Kropec A, Engels I, Daschner F. In vitro susceptibility of methicillin-resistant Staphylococcus aureus and slime-producing and non-slime producing coagulasenegative staphylococci to fusidic acid. Chemotherapy 1992; 38: $206-210$

5. Drugeon HB, Caillon J, Juvin ME. In-vitro antibacterial activity of fusidic acid alone and in combination with other antibiotics against methicillin-sensitive and -resistant Staphylococcus aureus. J Antimicrob Chemother 1994; 34: 899-907.

6. Coombs RRH. Fusidic acid in Staphylococcal bone and joint infection. J Antimicrob Chemother 1990; 25 Suppl B: 53-60.

7. Eykyn SJ. Staphylococcal bacteraemia and endocarditis and fusidic acid. J Antimicrob Chemother 1990; 25 Suppl B: 33-38.

8. Chopra I. Mechanisms of resistance to fusidic acid in Staphylococcus aureus. J Gen Microbiol 1976; 96: 229-238.

9. O'Brien FG, Botterill CI, Endersby TG, Lim RLG, Grubb WB, Gustafson JE. Heterogeneous expression of fusidic acid resistance in Staphylococcus aureus with plasmid or chromosomally encoded fusidic acid resistance genes. Pathology 1998; 30: $299-303$

10. Lacey RW, Rosdahl VT. An unusual 'penicillinase plasmid' in Staphylococcus aureus: evidence for its transfer under natural conditions. J Med Microbiol 1974; 7: 1-9.

11. Lacey RW, Grinsted J. Linkage of fusidic acid resistance to the pencillinase plasmid in Staphylococcus aureus. J Gen Microbiol 1972; 73: 501-508.

12. Courvalin P, Fiandt M. Aminoglycoside-modifying enzymes of
Staphylococcus aureus; expression in Esherichia coli. Gene 1980; 9: 247-269.

13. Sinden D, Chopra I. Fusidic acid resistance in Staphylococcus aureus. Zentalbl Bakteriol 1981; Suppl 10: 571-574.

14. Faber M, Rosdahl VT. Susceptibility of fusidic acid among Danish Staphylococcus aureus strains and fusidic acid consumption. J Antimicob Chemother 1990; 25 Suppl B: 7-14.

15. Toma E, Barriault D. Antimicrobial activity of fusidic acid and disk diffusion susceptibility testing criteria for gram-positive cocci. J Clin Microbiol 1995; 33: 1712-1715.

16. Shanson DC. Clinical relevance of resistance to fusidic acid in Staphylococcus aureus. J Antimicrob Chemother 1990; 25 Suppl B: 15-21.

17. Gransden WR, Eykyn SJ, Phillips I. Staphylococcus aureus bacteraemia: 400 episodes in St. Thomas's Hospital. BMJ 1984; 288: 300-303.

18. Turnidge J, Lawson P, Munro R, Benn R. A national survey of antimicrobial resistance in Staphylococcus aureus in Australian hospitals. Med J Aust 1989; 150: 65-72.

19. Turnidge JD, Nimmo GR, Francis G. Evolution of resistance in Staphylococcus aureus in Australian teaching hospitals. Med $J$ Aust 1996; 164: 68-71.

20. Riley TV, Pearman JW, Rouse IL. Changing epidemiology of methicillin-resistant Staphylococcus aureus in Western Australia. Med J Aust 1995; 163: 412-414.

21. Zaman R, Dibb WL. Methicillin-resistant Staphylococcus aureus (MRSA) isolated in Saudi Arabia: epidemiology and antimicrobial resistance patterns. J Hosp Infect 1994; 26: 297-300.

22. Torvaldsen S, Riley T. Emerging sodium fusidate resistance in Western Australian methicillin-resistant Staphylococcus aureus. Comm Dis Intel 1996; 20: 492-494.

23. Lang S, Raymond N, Brett M. Mupirocin-resistant S. aureus in Auckland. N Z Med J 1992; 105: 438.

24. Struelens MJ. Tracking the epidemiology of antimicrobial drug resistance in hospitals: time to deploy molecular typing. $J \mathrm{Med}$ Microbiol 1998; 47: 1035-1036.

25. National Committee for Clinical Laboratory Standards. Performance standards for antimicrobial disk susceptibility tests. Wayne, PA, NCCLS. 1998.

26. A guide to sensitivity testing. Report of the Working Party on Antibiotic Sensitivity Testing of the British Society for Antimicrobial Chemotherapy. J Antimicrob Chemother 1991; 27 Suppl D: 1-50.

27. Hamilton-Miller JMT, Maple PAC. Antibiogram typing of methicillin-resistant Staphylococcus aureus: a comparison with phage typing, biotyping and API Staph. Int J Med Microbiol Virol Parasitol Infect Dis 1993; 279: 214-224.

28. Townsend DE, Ashdown N, Bolton S, Grubb WB. The use of cetyltrimethylammonium bromide for the rapid isolation from Staphylococcus aureus of relaxable and non-relaxable plasmid DNA suitable for in vitro manipulation. Lett Appl Microbiol 1985; 1: 87-94.

29. Udo EE, Grubb WB. Genetic analysis of methicillin-resistant Staphylococcus aureus from a Nigerian hospital. J Med Microbiol 1993; 38: 203-208.

30. Tenover FC, Arbeit RD, Goering RV et al. Interpreting chromosomal DNA restriction patterns produced by pulsedfield gel electrophoresis: criteria for bacterial strain typing. J Clin Microbiol 1995; 33: 2233-2239. 